\title{
Characteristics and Mechanisms of Silicon-Oxide-Based Resistance Random Access Memory
}

\author{
Kuan-Chang Chang, Tsung-Ming Tsai, Ting-Chang Chang, Senior Member, IEEE, Hsing-Hua Wu, Jung-Hui Chen, \\ Yong-En Syu, Geng-Wei Chang, Tian-Jian Chu, Guan-Ru Liu, Yu-Ting Su, Min-Chen Chen, Jhih-Hong Pan, \\ Jian-Yu Chen, Cheng-Wei Tung, Hui-Chun Huang, Ya-Hsiang Tai, Der-Shin Gan, and Simon M. Sze
}

\begin{abstract}
Traditionally, a large number of silicon oxide materials are extensively used as various dielectrics for semiconductor industries. In general, silicon oxide cannot be used as resistance random access memory (RRAM) due to its insulating electrical properties. In this letter, we have successfully produced resistive switching and forming-free behaviors by zinc doped into silicon oxide. The current-voltage fitting data show that current transport mechanism is governed by Poole-Frenkel behavior in highresistance state and Ohm's law in low-resistance state, consisting with filament theory. Additionally, good endurance and retention reliabilities are exhibited in the zinc-doped silicon oxide RRAM.
\end{abstract}

Index Terms-Filament, resistive switch, silicon oxide, $\mathbf{Z n}$.

\section{INTRODUCTION}

$\mathbf{R}$ ECENTLY, modern semiconductor nonvolatile memories (NVMs) have been scaled constantly to achieve large capacity while device features approach the sub-100-nm regime to store a large amount of information. Nevertheless, for conventional charge-storage-based memories [1]-[4], the increasing demand for device densities by scaling dimension is expected to be a major challenge due to the technical and physical limitations. Resistance random access memory (RRAM) is one of the promising candidates for next-generation NVMs, due to its simple cell structure, low operating voltage, high operating speed, and nondestructive readout [5]-[10].

Manuscript received December 11, 2012; revised December 24, 2012; accepted December 29, 2012 Date of publication February 14, 2013; date of current version February 20, 2013. This work was supported by the National Science Council of the Republic of China under Contracts NSC-101-2120-M110-002 and NSC 101-2221-E-110-044-MY3. The review of this letter was arranged by Editor M. Jurczak.

K.-C. Chang, T.-M. Tsai, T.-J. Chu, J.-H. Pan, C.-W. Tung, H.-C. Huang, and D.-S. Gan are with the Department of Materials and Optoelectronic Science, National Sun Yat-Sen University, Kaohsiung 804, Taiwan.

T.-C. Chang, Y.-E. Syu, G.-R. Liu, Y.-T. Su, M.-C. Chen, and J.-Y. Chen are with the Department of Physics, National Sun Yat-Sen University, Kaohsiung 804, Taiwan, and also with the Advanced Optoelectronics Technology Center, National Cheng Kung University, Tainan 701, Taiwan (e-mail: tcchang@mail. phys.nsysu.edu.tw).

H.-H. Wu, G.-W. Chang, and Y.-H. Tai are with the Department of Photonics and the Institute of Electro-Optical Engineering, National Chiao Tung University, Hsinchu 300, Taiwan.

J.-H. Chen is with the Department of Chemistry, National Kaohsiung Normal University, Kaohsiung 82444, Taiwan (e-mail: t1446@nknucc.nknu.edu.tw).

S. M. Sze is with the Department of Physics, National Sun Yat-Sen University, Kaohsiung 804, Taiwan, and also with the Department of Electronics Engineering, National Chiao Tung University, Hsinchu 300, Taiwan (e-mail: simonsze@faculty.nctu.edu.tw).

Color versions of one or more of the figures in this letter are available online at http://ieeexplore.ieee.org.

Digital Object Identifier 10.1109/LED.2013.2241725
For virgin RRAM devices [11], [12], an initial "forming" stage is needed to break down the resistance switching layer until, ultimately, devices present resistive switching behaviors. However, the voltage required for forming process can be relatively high, which not only imposes severe constraint from a circuit design perspective but also affects the endurance behavior of the memory device due to substantial trap generation during forming process. It is, therefore, necessary to develop an RRAM device that can be operated without "forming" process to enhance the performance metrics of the RRAM circuit.

Zinc element is a popular material applied to $\mathrm{ZnO}$ nanostructure [13] and IGZO TFT devices which can be used for flatpanel display [14]. Therefore, zinc metal doped into $\mathrm{SiO}_{2}$ by cosputtering at room temperature was taken as the resistance switching layer of forming-free RRAM for the applications of system on panel. In addition, the conduction mechanism and material analyses were discussed to explain the influence of zinc metal doped in silicon oxide on forming-free resistive switching behaviors.

\section{EXPERIMENTAL SETUP}

First, a 30-nm $\mathrm{Zn}: \mathrm{SiO}_{x}$ thin film was deposited on a TiN/Ti/ $\mathrm{SiO}_{2} / \mathrm{Si}$ substrate by cosputtering with pure $\mathrm{SiO}_{2}$ and $\mathrm{Zn}$ targets using an RF power of $200 \mathrm{~W}$ and a dc power of $10 \mathrm{~W}$, respectively, in $\mathrm{Ar}=30 \mathrm{sccm}$ gas ambient with a working pressure of 4 mtorr. A Pt top electrode was deposited on the $\mathrm{Zn}: \mathrm{SiO}_{x}$ film to form $\mathrm{Pt} / \mathrm{Zn}: \mathrm{SiO}_{x} / \mathrm{TiN}$ sandwich structures. On the other hand, $\mathrm{Pt} / \mathrm{SiO}_{2} / \mathrm{TiN}$ sandwich structures were made by the same procedure as control samples. Finally, electrical device cells with a diameter of $4 \mu \mathrm{m}$ were fabricated through lithography and lift-off techniques and measured by an Agilent B1500 semiconductor parameter analyzer.

\section{Results AND Discussion}

Fig. 1(a) shows bipolar resistance switching characteristics of the $\mathrm{Pt} / \mathrm{Zn}: \mathrm{SiO}_{x} / \mathrm{TiN}$ device for dc voltage sweep operations. The current-voltage $(I-V)$ properties of the sputtered $\mathrm{SiO}_{2}$ layer cannot exhibit the RRAM properties due to its superior insulating properties shown in the lower right inset in Fig. 1(a). The voltage sweep bias was applied on the TiN electrode with the grounded Pt electrode shown in the lower left inset in Fig. 1(a). In particular, the device can exhibit resistive switching behavior without forming process, which is different to most of RRAM devices. In the initial state, the resistance state of 

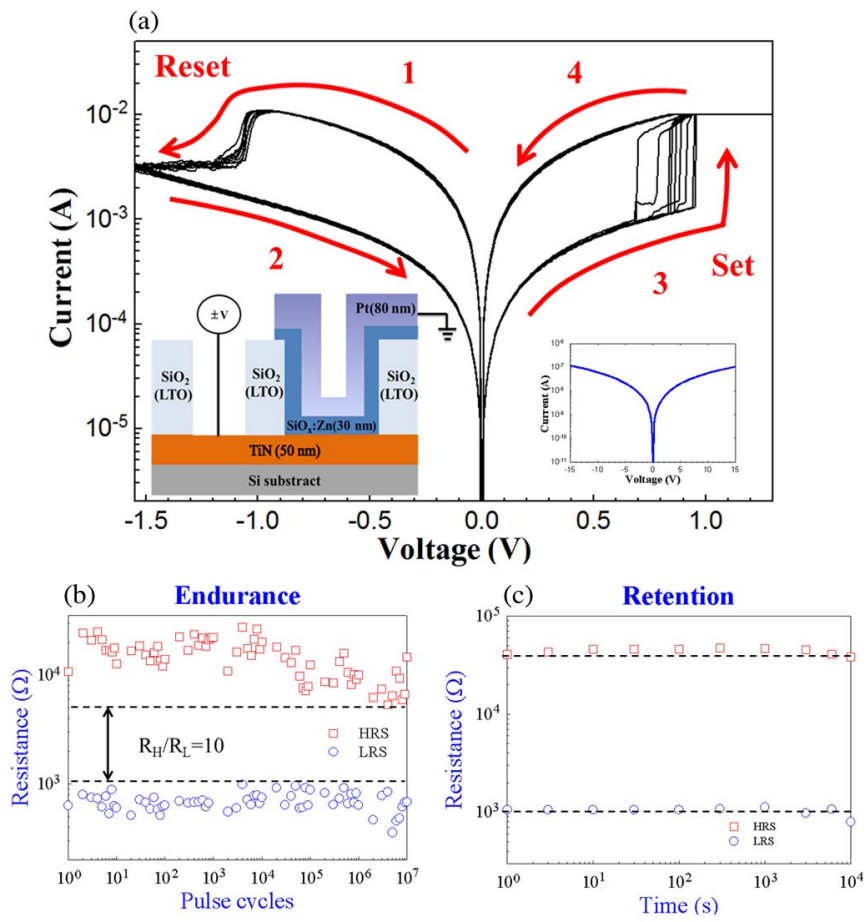

Fig. 1. (a) Bipolar resistance switching current-voltage $(I-V)$ curves of the $\mathrm{Pt} / \mathrm{Zn}: \mathrm{SiO}_{x} / \mathrm{TiN}$ device with compliance current of $10 \mathrm{~mA}$. The bottom left inset shows the schematic diagram measured with grounded Pt electrode. The bottom right inset shows the $I-V$ curve of the $\mathrm{Pt} / \mathrm{SiO}_{2} / \mathrm{TiN}$ device. (b) Endurance and (c) retention properties of $\mathrm{Pt} / \mathrm{Zn}: \mathrm{SiO}_{x} / \mathrm{TiN}$ memory device. Read voltage was $0.1 \mathrm{~V}$.

the $\mathrm{Zn}: \mathrm{SiO}_{x}$ device was at low-resistance state (LRS). As the voltage was swept from 0 to $-1.6 \mathrm{~V}$ without current compliance, an abrupt decrease in current was observed, where the cell switches to high-resistance state (HRS) from LRS, called as "reset process." In contrast, by sweeping the voltage from 0 to $1.3 \mathrm{~V}$ with a $10-\mathrm{mA}$ current compliance, the resistance switched from HRS to LRS, called as "set process." Moreover, the programming current of the device can be complied by integrating a selecting transistor.

To further investigate the reliability properties of the NVM device, endurance and retention tests were evaluated in Fig. 1(b) and (c), respectively. For endurance tests, the HRS was switched to the LRS by applying a set voltage of $1.5 \mathrm{~V}$ for $10 \mathrm{~ns}$, while the LRS was reset to the HRS by applying a reset voltage of $-2 \mathrm{~V}$ for $15 \mathrm{~ns}$. This resistive switching speed is competing with the operation speed of static random access memory. Both HRS and LRS resistance values were extracted at $0.1 \mathrm{~V}$. The resistance ratio between the HRS and the LRS was about ten times and can be maintained during the $10^{7}$ cycling bias pulse operations. The retention performance of the memory device was evaluated at $85^{\circ} \mathrm{C}$ by measuring the resistance values of the LRS and HRS at $0.1 \mathrm{~V}$ with an interval. The resistance values of LRS and HRS remain almost constant even after $10^{4} \mathrm{~s}$. This implicates that the resistance state did not vary easily due to oxygen diffusion under retention test.

The HRS and the LRS of $I-V$ curves were analyzed for the current conduction mechanisms to further discuss the resistance switching mechanisms in the $\mathrm{Zn}: \mathrm{SiO}_{x}$ thin film (Fig. 2). The blue-square symbol in the $I-V$ curve reveals that the current conduction in the HRS was dominated by the Poole-Frenkel conduction mechanism, followed by Ohm's law with the square

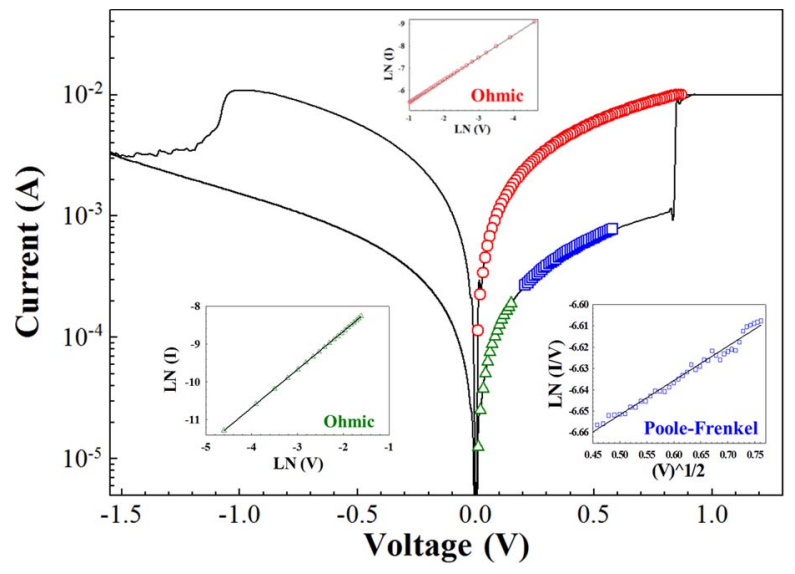

Fig. 2. $I-V$ curve fitting of $\mathrm{Pt} / \mathrm{Zn}: \mathrm{SiO}_{x} / \mathrm{TiN}$ memory device with various carrier transport mechanisms in different bias regions and resistance states.

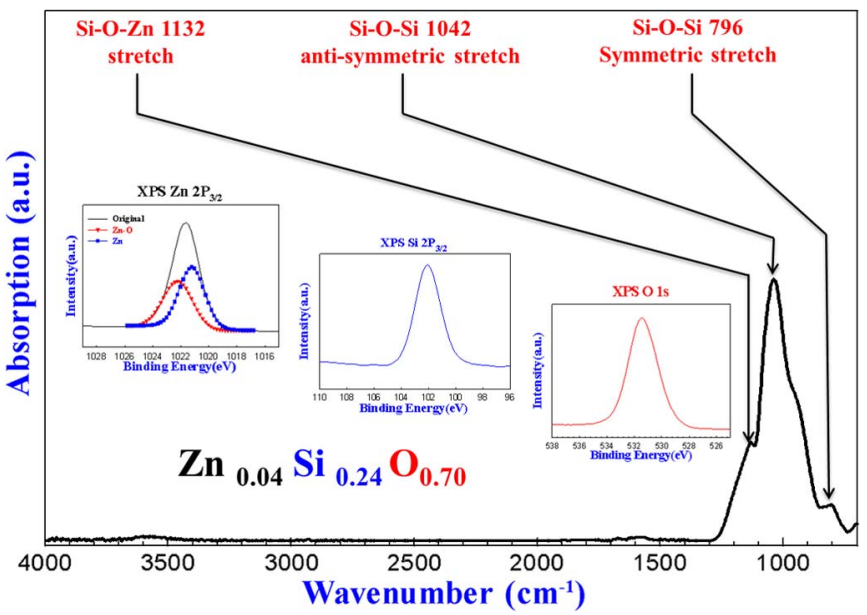

Fig. 3. FTIR spectra of $\mathrm{Zn}: \mathrm{SiO}_{x}$ film measured in middle infrared region. The XPS spectra of $\mathrm{Zn} 2 P_{3 / 2}$, Si $2 P_{3 / 2}$, and O $1 s$ core levels in $\mathrm{Zn}: \mathrm{SiO}_{x}$ film are shown in the insets.

root of the applied voltage at high fields (the lower right inset in Fig. 2). The ohmic conduction occurred at the green-trianglemarked region in the HRS due to intrinsic carrier in the silicon oxide (the lower left inset in Fig. 2). Moreover, the current conduction in the LRS complied with the ohmic conduction mechanism.

To analyze the influence of $\mathrm{Zn}$ doping on resistance switching characteristics in silicon oxide thin films, Fourier transform infrared spectroscopy (FTIR) was used to investigate the chemical bonding of the $\mathrm{Zn}: \mathrm{SiO}_{x}$ film in this study. Fig. 3 shows that the $\mathrm{Si}-\mathrm{O}-\mathrm{Zn}$ stretch bonding was found in the $\mathrm{Zn}: \mathrm{SiO}_{x}$ film at $1132 \mathrm{~cm}^{-1}$. In addition, the antisymmetric stretch mode and the symmetric stretch mode of $\mathrm{Si}-\mathrm{O}-\mathrm{Si}$ bonds were discovered at 1042 and $796 \mathrm{~cm}^{-1}$, respectively. According to these absorption peaks expressed in FTIR spectrums, we can confirm that the $\mathrm{Zn}$ element was bonded with the oxygen element in the silicon oxide film. To analyze the chemical composition of the $\mathrm{Zn}: \mathrm{SiO}_{x}$ film in this study, X-ray photoelectron spectroscopy (XPS) of $\mathrm{Zn} 2 P_{3 / 2}$, Si $2 P_{3 / 2}$, and $\mathrm{O} 1 s$ peaks was performed, and the XPS spectra are shown in the insets in Fig. 3. The mole fraction of $\mathrm{Zn}: \mathrm{Si}: \mathrm{O}$ in the $\mathrm{Zn}: \mathrm{SiO}_{x}$ film was $4.9 \%: 24.9 \%: 70.2 \%$ calculated from the peak areas of $\mathrm{Zn}, \mathrm{Si}$, and O XPS spectra. In addition, compared with the areas of deconvolution peaks of $\mathrm{Zn} 2 P_{3 / 2}$ core levels, we found that the mole fraction of 


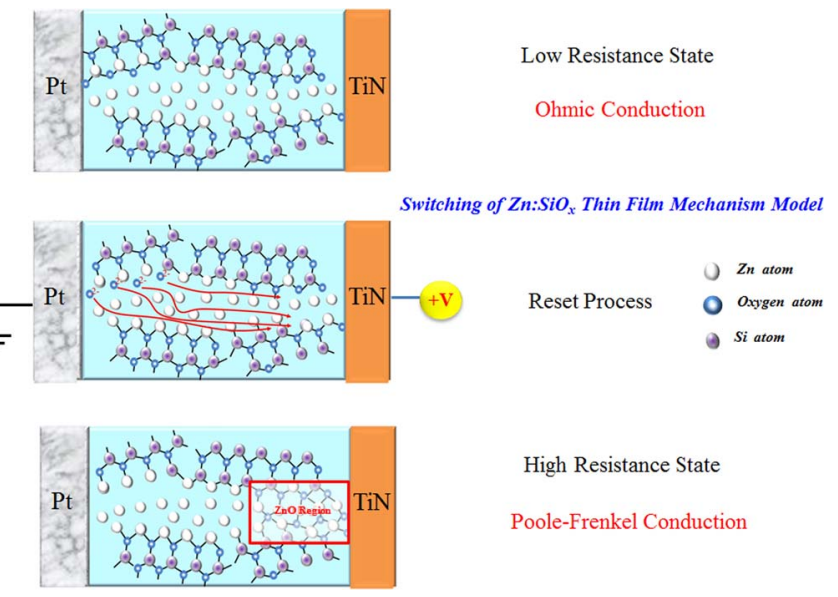

Fig. 4. Schematic diagram of reaction model in $\mathrm{Zn}: \mathrm{SiO}_{x}$ film during resistive switching operation of the device.

$\mathrm{ZnO}: \mathrm{Zn}$ was $45.1 \%: 54.9 \%$ in the $\mathrm{Zn}: \mathrm{SiO}_{x}$ film. The results of FTIR and XPS analyses demonstrated that the zinc metal is contained in the $\mathrm{Zn}: \mathrm{SiO}_{x}$ film and the $\mathrm{Si}-\mathrm{O}-\mathrm{Zn}$ stretch bonding is formed on the boundary of zinc metal and silicon oxide.

Based on the electrical and material analyses, we propose a reaction model to explain the resistive switching behavior of Zn-doped silicon-oxide-based RRAM in Fig. 4. As the sample was fabricated to the $\mathrm{Pt} / \mathrm{Zn}: \mathrm{SiO}_{x} / \mathrm{TiN}$ device, the zinc element was wrapped by silicon oxide, leading to the zinc conductive filament connecting with the two electrodes. Therefore, the electrical current is dominated by ohmic conduction in LRS of the device. Then, the conductive current causes the negative oxygen ion surrounding the conductive filament close to the Pt electrode to drift to the TiN electrode, oxidizing the zinc element to zinc oxide $(\mathrm{ZnO})$. Hence, the electrical current will be obstructed by the $\mathrm{ZnO}$ region shown in Fig. 4, which will make the electrical current transfer to HRS in the RRAM device. In virtue of the boundary of $\mathrm{ZnO}$ and silicon oxide existing strained bonds, the conduction mechanism in HRS of the device complied with the Poole-Frenkel conduction [15].

\section{CONCLUSION}

In conclusion, the reproducible forming-free bipolar resistance switching characteristics have been achieved by doping $\mathrm{Zn}$ metal into silicon oxide film using cosputtering technique at room temperature. According to electrical and material analyses, the current transport mechanism of LRS in $\mathrm{Zn}: \mathrm{SiO}_{x}$ RRAM was governed by ohmic conduction due to the conductive filament. Additionally, the current transport mechanism of HRS in $\mathrm{Zn}: \mathrm{SiO}_{x}$ RRAM was governed by Poole-Frenkel conduction, which was attributed to the strained bonds at the boundary of $\mathrm{ZnO}$ and silicon oxide nearby the TiN electrode. Based on the reliability evaluation, the $\mathrm{Zn}: \mathrm{SiO}_{x}$ RRAM satisfied the demand of modern NVMs.

\section{ACKNOWLEDGMENT}

This work was performed at the National Science Council Core Facilities Laboratory for Nano-Science and NanoTechnology in the Kaohsiung-Pingtung area.

\section{REFERENCES}

[1] T. C. Chang, F. Y. Jian, S. C. Chen, and Y. T. Tsai, "Developments in nanocrystal memory," Mater. Today, vol. 14, no. 12, pp. 608-615, Dec. 2011

[2] F. M. Yang, T. C. Chang, P. T. Liu, P. H. Yeh, Y. C. Yu, J. Y. Lin, S. M. Sze, and J. C. Lou, "Memory characteristics of Co nanocrystal memory device with $\mathrm{HfO}_{2}$ as blocking oxide," Appl. Phys. Lett., vol. 90, no. 13, pp. 132102-1-132102-3, Mar. 2007.

[3] J. Liu, Q. Wang, S. B. Long, M. H. Zhang, and M. Liu, "Metal/ $/ \mathrm{Al}_{2} \mathrm{O}_{3} /$ $\mathrm{ZrO}_{2} / \mathrm{SiO}_{2} / \mathrm{Si}$ (MAZOS) structure for high-performance non-volatile memory application," Semicond. Sci. Technol., vol. 25, no. 5, p. 055013, May 2010.

[4] F. M. Yang, T. C. Chang, P. T. Liu, U. S. Chen, P. H. Yeh, Y. C. Yu, J. Y. Lin, S. M. Sze, and J. C. Lou, "Nickel nanocrystals with $\mathrm{HfO}_{2}$ blocking oxide for nonvolatile memory application," Appl. Phys. Lett., vol. 90, no. 22, pp. 222104-1-222104-3, May 2007.

[5] K. C. Chang, T. M. Tsai, T. C. Chang, Y. E. Syu, S. L. Chuang, C. H. Li, D. S. Gan, and S. M. Sze, "The effect of silicon oxide based RRAM with tin doping," Electrochem. Solid-State Lett., vol. 15, no. 3, pp. H65-H68, Dec. 2011.

[6] Y. E. Syu, T. C. Chang, T. M. Tsai, Y. C. Hung, K. C. Chang, M. J. Tsai, M. J. Kao, and S. M. Sze, "Redox reaction switching mechanism in RRAM device with $\mathrm{Pt} / \mathrm{CoSiO}_{\mathrm{X}} / \mathrm{TiN}$ structure," IEEE Electron Device Lett., vol. 32, no. 4, pp. 545-547, Apr. 2011.

[7] K. C. Chang, T. M. Tsai, T. C. Chang, Y. E. Syu, C. C. Wang, S. L. Chuang, C. H. Li, D. S. Gan, and S. M. Sze, "Reducing operation current of Ni-doped silicon oxide resistance random access memory by supercritical $\mathrm{CO}_{2}$ fluid treatment," Appl. Phys. Lett., vol. 99, no. 26, pp. 263501-1-263501-4, Dec. 2011.

[8] Q. Liu, C. M. Dou, Y. Wang, S. B. Long, W. Wang, M. Liu, M. H. Zhang, and J. N. Chen, "Formation of multiple conductive filaments in the $\mathrm{Cu} / \mathrm{ZrO}_{2}$ : $\mathrm{Cu} / \mathrm{Pt}$ device," Appl. Phys. Lett., vol. 95, no. 2, pp. 023501-1-023501-3, Jul. 2009.

[9] S. Zhang, S. B. Long, W. H. Guan, Q. Liu, Q. Wang, and M. Liu, "Resistive switching characteristics of MnOx-based ReRAM," J. Phys. D, Appl. Phys., vol. 42, no. 5, p. 055112, Mar. 2009.

[10] Y. Wang, Q. Liu, S. B. Long, W. Wang, Q. Wang, M. H. Zhang, S. Zhang, Y. T. Li, Q. Y. Zuo, J. H. Yang, and M. Liu, "Investigation of resistive switching in $\mathrm{Cu}$-doped $\mathrm{HfO}_{2}$ thin film for multilevel non-volatile memory applications," Nanotechnology, vol. 21, no. 4, p. 045202, Jan. 2010.

[11] R. Huang, L. J. Zhang, D. J. Gao, Y. Pan, S. Q. Qin, P. R. Tang, Y. M. Cai, and Y. Y. Wang, "Resistive switching of silicon-rich-oxide featuring high compatibility with CMOS technology for 3D stackable and embedded applications," Appl. Phys. A, vol. 102, no. 4, pp. 927-931, Apr. 2011.

[12] C. Y. Liu and P. W. Sung, "Different resistive switching characteristics of a $\mathrm{Cu} / \mathrm{SiO}_{2} / \mathrm{Pt}$ structure," Jpn. J. Appl. Phys. 1, Regul. Rap. Short Notes, vol. 50, no. 9, pp. 091101-1-091101-4, Sep. 2011.

[13] K. C. Chang, T. M. Tsai, T. C. Chang, Y. E. Syu, H. C. Hung, Y. C. Hung, T. F. Young, D. S. Gan, and N. J. Ho, "Low-temperature synthesis of ZnO nanotubes by supercritical $\mathrm{CO}_{2}$ fluid treatment," Electrochem. Solid-State Lett., vol. 14, no. 9, pp. K47-K50, June 2011.

[14] T. C. Chen, T. C. Chang, C. T. Tsai, T. Y. Hsieh, S. C. Chen, C. S. Lin, M. C. Hung, C. H. Tu, J. J. Chang, and P. L. Chen, "Behaviors of InGaZnO thin film transistor under illuminated positive gate-bias stress," Appl. Phys. Lett., vol. 97, no. 11, pp. 112104-1-112104-3, Sep. 2010.

[15] S. Habermehl and C. Carmignani, "Correlation of charge transport to local atomic strain in Si-rich silicon nitride thin films," Appl. Phys. Lett., vol. 80, no. 2, pp. 261-263, Jan. 2002. 